\title{
PROTON BUNCH COMPRESSION STRATEGIES ${ }^{1}$
}

\author{
VALERI LEBEDEV ${ }^{\dagger}$ \\ Fermilab, P.O. Box 500 \\ Naperville, IL 60563, USA
}

\begin{abstract}
The paper discusses main limitations on the bea $\mathrm{m}$ power and othe $\mathrm{r}$ machine parameters for a 4 MW proton drive $\mathrm{r}$ for $\mathrm{m}$ uon collider. The strongest limitation comes fr om a longitudinal microwave instability limiting the beam power to about $1 \mathrm{MW}$ for an $8 \mathrm{GeV}$ compressor ring.
\end{abstract}

\section{Introduction}

The muon collider luminosity is proportional to the square of the number of muons delivered to a collider and consequently to the square of proton driver power. In the energy range of 8 to $20 \mathrm{G} \mathrm{eV}$ the efficiency of muon production per unit power of the proton beam has a weak dependence on the beam energy and therefore the major re quirement for the proton driver is the total be am power. Presently, there are a few proposals for a muon collider [1]. All of them require a proton driver with $4 \mathrm{MW}$ beam power and the rms bunch length at the muon production target below 2-3 ns. While the repetition ra tes of different proposals vary, in this paper we assume the repetition rate of $15 \mathrm{~Hz}$.

At the very least the proton part of the muon collider consists of two pieces. The fi rst one $\mathrm{i} s \mathrm{t}$ he prot on dri ver whi ch $\mathrm{c}$ an be based on a rapid cycling synchrotron (RCS) or supe rconducting (SC ) 1 inac. The se cond one i s a compressor ring. In order to simplify the requirements of the RF system some proposals suggest an intermediate ring to accumulate the strip-injected $\mathrm{H}^{-}$beam accelerated in the SC lin ac - the A ccumulator [2]. In this paper we do not discuss li mitations com ing from the bea $\mathrm{m}$ i njection and RF systems and therefore do not separate the Accumulator and C ompressor rings. In thi s case the process c onsists of $t$ wo major st eps: (1) the injection of the be am to the compressor ring, and (2) the longitudinal bunch compression performed by the

\footnotetext{
${ }^{1 *}$ This work is supported by the U.S. De partment of E nergy under c ontract N o. D E-AC02-07CH11359

† val@fnal.gov
} 
bunch rotation in the phase space excided by high voltage RF system.

The main beam power limitations $\mathrm{c}$ ome from the longitudinal microwave instability, the betatron tune shift due to bea $\mathrm{m}$ space charge and the beam size limitation on the target. There are al so a number of engi neering limitations which are also important but will not be discussed in here in any detail.

\section{Longitudinal Microwave Instability}

First, we consider the beam stability criterion for the continuous beam. Let the bea $\mathrm{m} 1$ ongitudinal dist ribution be desc ribed by function $f(p)$ whi ch i s normalized to unity:

$$
\int f(p) d p=1,
$$

where $p$ is a momentum devi ation from its mean val ue, $p_{0}$. We a dditionally rescale the argument of the distribution function by the rms momentum spread so that the new and old distributions are related as following:

$$
f_{0}(p)=\frac{1}{\sigma_{p}} \psi_{0}\left(\frac{p}{\sigma_{p}}\right), \quad \sigma_{p}^{2}=\int p^{2} f_{0}(p) d p \text {. }
$$

This results in the following properties of the new distribution function:

$$
\int \psi_{0}(x) d x=1 \quad, \quad \int x^{2} \psi_{0}(x) d x=1
$$

where $x=p / \sigma_{p}$.

The coherent frequency, $\delta \omega$, of the mode $n \mathrm{c}$ an be found from the wellknown dispersion equation [3]:

$$
1-i A_{n} \int_{\delta \rightarrow+0} \frac{d \psi_{0} / d x}{y+x-i \delta \operatorname{sign}(n)} d x=0
$$

where

$$
A_{n} \equiv \frac{e I_{0}}{2 \pi c p_{0} \beta \eta}\left(\frac{p_{0}}{\sigma_{p}}\right)^{2}\left(\frac{Z_{n}}{n}\right), \quad y=\frac{\delta \omega}{n \omega_{0} \eta}\left(\frac{p_{0}}{\sigma_{p}}\right),
$$

$I_{0}$ is the beam current, $e$ is the particle charge, $Z_{n}$ is the longitudinal impedance on the $n$-th harmonic of the revolution frequency, $\eta=\alpha-1 / \gamma^{2}$ is the ring slip factor, and $c \beta$ is the beam velocity. At the stability boundary the imaginary part of coherent frequency is equal to zero, $\operatorname{Im}(\delta \omega)=0$. Using this condition in Eq. (4) one obtains a parametric equation for the stability boundary: 


$$
A(y)=\left(i \int_{\delta \rightarrow+0} \frac{d \psi_{0} / d x}{y+x-i \delta \operatorname{sign}(n)} d x=0\right)^{-1}
$$

Numerical i ntegration of Eq. (6) was carried out for the fol lowing set of functions:

$$
\psi_{k}(x)=\frac{1}{b_{n}} \exp \left(-\frac{x^{2 k}}{s_{k}}\right),
$$

where co efficients $b_{k}$ an $d s_{k}$ are det ermined by Eqs . (3). F or the fi rst four functions, $k=1 \ldots 4$, they are:

$$
\begin{aligned}
& b_{k}=\left\{\sqrt{2 \pi}, \frac{\pi^{3 / 2}}{\sqrt[4]{2}(\Gamma(3 / 4))^{2}}, \frac{2^{3 / 2} \pi^{5 / 4}}{3(\Gamma(5 / 6))^{3 / 2}}, \frac{(\Gamma(1 / 8))^{3 / 2}}{4 \sqrt{\Gamma(3 / 8)}}\right\} \approx\{2.507,3.118,3.288,3.358\}, \\
& s_{k}=\left\{2, \frac{\sqrt{2} \pi}{(\Gamma(3 / 4))^{2}},\left(\frac{2 \sqrt{\pi}}{\Gamma(5 / 6)}\right)^{3},\left(\frac{(\Gamma(1 / 8))}{\Gamma(3 / 8)}\right)^{4}\right\} \approx\{2,8.754,30.97,102.04\} .
\end{aligned}
$$

The function $\psi_{1}(x)$ describes the Gaussian distribution. Figure 1 present s plots for the first four functions and corresponding stability boundaries.
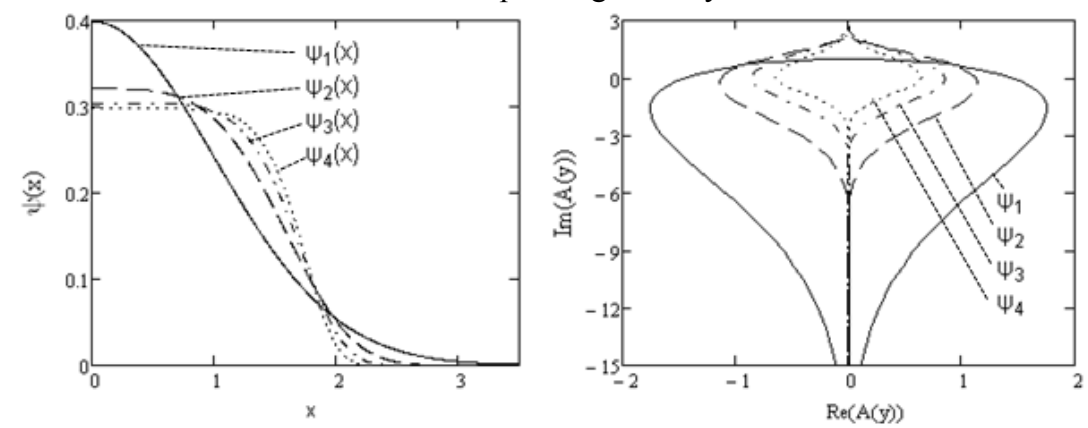

Figure 1. Plots for functions $\psi_{k}(x)$ (left) and corresponding stability boundaries (right).

In the ene rgy range necessary for the prot on drive $r t$ he impedance is dominated by $\mathrm{t}$ he bea $\mathrm{m}$ spa ce $\mathrm{c}$ harge and $Z \propto i$. C onsequently, be low the transition $(\eta<0) A_{n} \propto Z \eta \propto-i$. One can see from the Figure 1 that for Gaussian beam $(k=1)$ the sta bility regi on bel ow transition is signi ficantly hi gher than above. How ever this difference is $\mathrm{m}$ uch less significant for distributions with steeper edge $(k>1)$. Note that these distributions better represent a longitudinal distribution obtained by painting small emittance linac beam into a much larger ring acc eptance. Conse quently, ope ration above c ritical ene rgy 1 ooks $\mathrm{m}$ ore preferable because significantly larger value of slip factor can be achieved. In 
this case the stability criterion is:

$$
\left|A_{n}\right| \equiv\left|\frac{e I_{0}}{2 \pi c p_{0} \beta \eta}\left(\frac{p_{0}}{\sigma_{p}}\right)^{2}\left(\frac{Z_{n}}{n}\right)\right| \leq B_{k} \quad, \text { (9) }
$$

For the first four functions $\psi_{k}(x)$ the coefficients $B_{k}$ are: $B_{k}=\{1,2.189,2.665$. 2.747 (these numbers correspond to $y$ coordinates of top points at the stability diagram of Figure 1.) In further consideration we will assume $B_{k} \approx 2$.

The space charge impedance for the round beam with Gaussian distribution with rms size $\sigma_{\perp}$ in the round vacuum chamber of radius $a$ is

$$
\frac{Z_{n}}{n} \approx i \frac{Z_{0}}{\beta \gamma^{2}} \ln \left(\frac{a}{1.5 \sigma_{\perp}}\right),
$$

where $Z_{0} \approx 377 \Omega$ is the impedance of free space, and $\beta$ and $\gamma$ are the relativistic factors. In the energy range of 8 to $20 \mathrm{GeV}\left|Z_{n} / n\right|$ is changing from $\sim 10$ to $\sim 2 \Omega$. An achievement of high bunch density implies good electrodynamics of vacuum chamber. In this case the impedances due to vacuum chamber inhomogeneities and a finite value of its wall resistivity do not exceed $1 \Omega$ for frequencies above the particle revolution frequency of $\sim 1$ MHz. Substituting Eq. (10) int o Eq. (9) and equaling $B_{k}=2$ results in the stability condition:

$$
\frac{r_{p} N}{\beta^{2} \gamma^{3} \eta\left(\sigma_{p} / p\right)^{2} L_{b}} \ln \left(\frac{a}{1.5 \sigma_{\perp}}\right) \leq 1 \quad,(11)
$$

where $r_{p}$ is the classical proton radius, $N$ is the number of particles in the beam and $L_{b}$ is the bunch length. Here we imply the uniform particle density along the bunch. It happens naturally if the beam is adiabatically debunched after arriving from the RCS. A s can be se en from Eq. (11) the stability th reshold does not depend on the mode number because the longitudinal impedance, $Z_{n} / n$, does not change up to very high frequency, $f_{\max } \approx c \gamma /(4 a)$. For the energy range of 8 to 20 $\mathrm{GeV}$ it corresponds to frequency range of $\sim 20-40 \mathrm{GHz}$. Above this frequency $Z_{n} / n$ starts to decrease.

Well above the instability threshold the instability growth rate can be easily calculated and is equal to:

$$
\lambda_{n} \approx n \omega_{0} \sqrt{\frac{2 r_{p} N \eta}{\beta^{2} \gamma^{3} L_{b}} \ln \left(\frac{a}{1.5 \sigma_{\perp}}\right)},
$$

where we assume that the space charge makes a dominant contribution to the longitudinal impedance. As one can see from the above equation the growth rate 
is proportional to the harmonic number and the instability can be extremely fast at the high frequenc y end. Although a low frequency part, $\leq 0.5 \mathrm{GHz}$, can in principle be stabilized by a longitudinal damper the high frequency part cannot be stabilized; and therefore one should not exceed the instability threshold. Note that Eq. (11) and (12) we re de rived for the cont inuous be am. Howe ver at sufficiently high frequencies, where $t$ he growth ra te bec omes la rger than the synchrotron fre quency, these equations correc tly de scribe $t$ he $t$ hreshold a nd growth rat e. In $t$ his high freque ncy re gion $t$ his inst ability i s called the microwave instability.

\section{Mitigation of the Microwave Instability}

Expressing the number of pa rticles through ot her $\mathrm{m}$ achine and be am parameters in Eq. (11) one obta ins the beam power limitation imposed by the longitudinal instability:

$$
P_{\max } \leq f_{\text {rep }} m_{p} c^{2}(\gamma-1) \frac{\beta^{2} \gamma^{3} \eta\left(\varepsilon_{L} / p_{0}\right)^{2}}{F_{\varepsilon}^{2} r_{p} L_{b} \ln \left(a /\left(1.5 \sigma_{\perp}\right)\right)},
$$

where $f_{\text {rep }}$ is the repetition rate, $m_{p}$ is the proton mass, $\varepsilon_{L}=F_{\varepsilon} \sigma_{p} L_{b}$ is the $95 \%$ beam 1 ongitudinal em ittance, and $F_{\varepsilon}$ is a form -factor binding up t he bunch length, the rms momentum spread and the longitudinal emittance. For the first four functions $\psi_{k}$ the form-factors are: $F_{\varepsilon} \approx\{1.96,1.80,1.75,1.71\}$.

The longi tudinal em ittance is approxim ately c onserved during the bunc $h$ rotation, and, as can be se en from Eq. (13), the beam power is limited by the initial bunch 1 ength, $L_{b}$. The bunch rot ation shortens t he bunch and, consequently, widens the stability margin. Note that the momentum acceptance of the compressor ring is m uch la rger th an the in itial momentum sp read and therefore the instability does not cause the beam loss. It rather results in a fast increase of the m omentum spread to its value de termined by Eq. (11). Consequently, the bunch rotation results in a longer bunch on the target.

The required value of longitudinal emittance is set by the rms bunch length on the target, $L_{t}$, and the maximum momentum spread, $\Delta p_{\max }$. The bunch length on the target is determined by requirements of the muon cooling channel and, as it wa s a lready quoted, is $\sim 60 \mathrm{~cm}$. The relative momentum spre ad is set by nonlinear chromatic effects in the compressor ring and the chromaticity of the beam focusing on the $t$ arget. In our considerations $w$ e wi 11 assum e t he momentum aperture $\pm 2.5 \%$ - sam e as for $\mathrm{t}$ he Debunc her $-\mathrm{t}$ he F ermilab ring with largest momentum and transverse acceptances. It results in $\varepsilon_{L} / c p_{0} \approx 90 \mathrm{ps,}$ which, in $\mathrm{t}$ he first a pproximation, doe s not depe nd on the compressor ring 
energy.

To maximize the slip factor a sufficiently small transition energy should be chosen. In this case $\eta \approx \alpha \approx D / R_{0}$, where $R_{0}$ is the mean ring radius and $D$ is the effective di spersion. On other hand $\Delta p_{\max } / p_{0} \approx a_{\max } / D$, w here $a_{\max }$ is the maximum beam size in the dipol e. Com bining one fina lly obt ains: $\eta \Delta p_{\max } / p_{0} \approx a_{\max } / R_{0}$. Taking this into ac count we can write a n est imate for power limitation resulting from Eq. (13) in the following form:

$$
P_{\max } \approx \frac{1}{2} \frac{f_{\text {rep }} m_{p} c^{2}(\gamma-1) \gamma^{3} \beta^{2} L_{t}{ }^{2} a_{\max }}{2 \pi r_{p} R_{0}{ }^{2} \eta_{\text {fill }} \ln (a /(1.5 \sigma))} \frac{\Delta p_{\max }}{p_{0}}
$$

where $\eta_{\text {fill }}=L_{b} /\left(2 \pi R_{0}\right)$ is the part of the machine circumference taken by the beam after injection, and the factor $1 / 2$ at the right-hand side corrects for real machine optics and difference between $\mathrm{rms}$ and maximum momentum spreads on the target (see details below). Note that in difference to Eq. (13) this equation is approximate and should be used cautiously. However it correctly reflects the dependence of bea $\mathrm{m}$ power on main machine and beam parameters. For an 8 $\mathrm{GeV}$ compressor ring with average radius $R_{0}=42 \mathrm{~m}$ and aperture $a_{\max }=10 \mathrm{~cm}$ one obtains $P_{\max } \approx 1.2 \mathrm{MW}$. O ne can see $\mathrm{t}$ hat the maximum power has very steep dependence on $\mathrm{t}$ he beam ene rgy. Taking into ac count that $R_{0} \propto \gamma$ and other parameters have comparatively weak dependence on the beam energy one obtains that the beam power is proportional to $\gamma^{2}$.

\section{Limitations on the Beam Emittance}

For a round Gaussian bea $\mathrm{m}$ and the smooth foc using a pproximation the betatron tune shift due to beam space charge is equal to:

$$
\delta v_{S C}=\frac{r_{p} R_{0}}{2 \beta^{2} \gamma^{3} \varepsilon_{\perp}} \frac{d N}{d s}(15)
$$

where $d N / d s$ is the bunch longitudinal density, and $\varepsilon_{\perp}$ is the transverse rms beam emittance. The tune shi $\mathrm{ft}$ ac hieves $\mathrm{i}$ ts maximum at he $\mathrm{e}$ d of bunc $\mathrm{h}$ compression and $\mathrm{i}$ mposes serious limitations on $\mathrm{t}$ he $\mathrm{m}$ achine parameters. Assuming a Gaussian longitudinal distribution at the end of bunch compression one can obtain the power limitation imposed by the tune shift:

$$
P_{\max } \leq f_{\text {rep }} m_{p} c^{2}(\gamma-1) \frac{2 \sqrt{2 \pi} \beta^{2} \gamma^{3} \varepsilon_{\perp} L_{t}}{r_{p} R_{0}} \delta v_{S C} .
$$

A conservative estimate based on the experience accumulated in Fermilab and other laboratories re quires $\delta v_{S C}$ be ing less or about 0.05 . In this case the 
beam loss is expected to be small - the primary requirement for a $\mathrm{M} \mathrm{W}$ scale machine. More detailed study is required to see a feasibility of larger tune shift achievement without an increase of the beam loss.

An in crease of the tr ansverse emitta nce is a natural way to mitigate the space charge effects. However this increase is limited by a necessity to focus the beam on the muon production target. An optimal rms beam size on the target is $2.5 \mathrm{~mm}$ [4]. The length of prot on beam int eraction wit $\mathrm{ht}$ he ta rget i s comparatively large. That li mits the bea $\mathrm{m}$ angul ar spread on the ta rget and, consequently, the beta-function on the target, $\beta^{*}$. The pion production suffers if $\beta^{*} \leq 20 \mathrm{~cm}$. That determines the maximum acceptable emittance to be $\sim 30 \mathrm{~mm}$ mrad i ndependently on $\mathrm{t}$ he be am energy. Taki ng i nto a ccount that $R_{0}$ is proportional to the beam momentum results in that $P_{\max }$ is proportional to $\sim \gamma^{3}$.

\section{Compressor Ring and its Tentative Parameters}

The beam power 1 imitations imposed by the microwave instability and by the betatron tune shift due to beam space charge become more stringent with an increase of average machine ra dius. Therefore the compressor ring considered here is built with high field superferic mag nets. That allows o ne to combine large magnetic field and a large radial aperture.

Table 1. Tentative machine and beam parameters.

\begin{tabular}{lc}
\hline Beam energy (kinetic) & $8 \mathrm{GeV}$ \\
Circumference 223 & $\mathrm{~m}$ \\
Number of particles & $5.2 \cdot 10^{13}$ \\
Duty factor at injection, $L_{b} / 2 \pi R_{0}$ & 0.2 \\
Repetition rate & $15 \mathrm{~Hz}$ \\
Beam power & $1 \mathrm{MW}$ \\
Transition energy, GeV & 2.45 \\
Betatron tunes & 5.59 \\
Dipole magnetic field & $3 \mathrm{~T}$ \\
Dipole length, m & 3.1 \\
Acceptance & $140 \mathrm{~mm} \mathrm{mrad}$ \\
Rms emittance & $8.8 \mathrm{~mm} \mathrm{mrad}$ \\
Space charge betatron tune shifts, $\delta v_{\mathrm{x}} / \delta v_{\mathrm{y}}$ & $0.057 / 0.087$ \\
Harmonic number & 1 \\
RF voltage for bunch rotation & $2.5 \mathrm{MV}$ \\
Duration of bunch rotation & $146 \mathrm{turns}$ \\
Rms momentum before rotation & $5.4 \cdot 10^{-4}$ \\
Longitudinal impedance, $Z_{n} / n$ & $10 \Omega$ \\
$Z_{n} / n$ at the stability boundary at injection & $20 \Omega$ \\
\hline
\end{tabular}

The compressor ring energy is chosen to be $8 \mathrm{GeV}$ to be matched to the energy of present Fermilab Booster as well as to the energy of new Fe rmilab 
proton sourc e w hich i s expe cted t o st art ope rations before 2020. The compressor ring represents a racetrack with FODO focusing and the dispersion zeroed in the two straight sections by missed dipoles. Figure 2 presents the betafunctions, the dispersion and the beam envelopes for a quarter of the ring and Table 1 presents the beam and machine parameters.

Figure 3 presents the longitudinal phase space and the longitudinal bunch density at the end of bunch rotation. To mitigate the microwave instability the bunch length before the rotation is short and, consequently, a voltage of the first RF harmonic creates sufficiently linear bunch rotation. Effect of the beam space charge on the rotation is also small. One can see that afte r rota tion the rms bunch length is $65 \mathrm{~cm}$ - close to the required value. The factor of $2 \mathrm{~m}$ argin for the microwave instability presented in Table 1 was computed for uniform initial longitudinal di stribution. H owever i $\mathrm{t}$ he st rip-injection i s used $\mathrm{t}$ he be am accumulation oc curs during $\mathrm{m}$ any hundre $\mathrm{ds}$ of $\mathrm{t}$ urns. Consequently, the synchrotron motion results in a particle density increase in the bunch center and reduction of the stability margin.
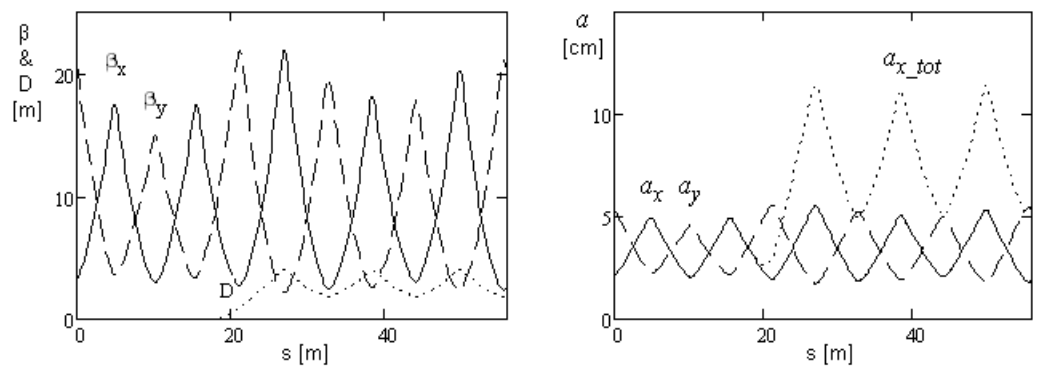

Figure 2. Twiss parameters (left) and beam envelopes (right) for a quarter of compressor ring. Beam envelopes are build for $\varepsilon_{x}=\varepsilon_{y}=140 \mathrm{~mm} \mathrm{mrad}(4 \sigma)$, and $\Delta p / p_{0}= \pm 0.025$.
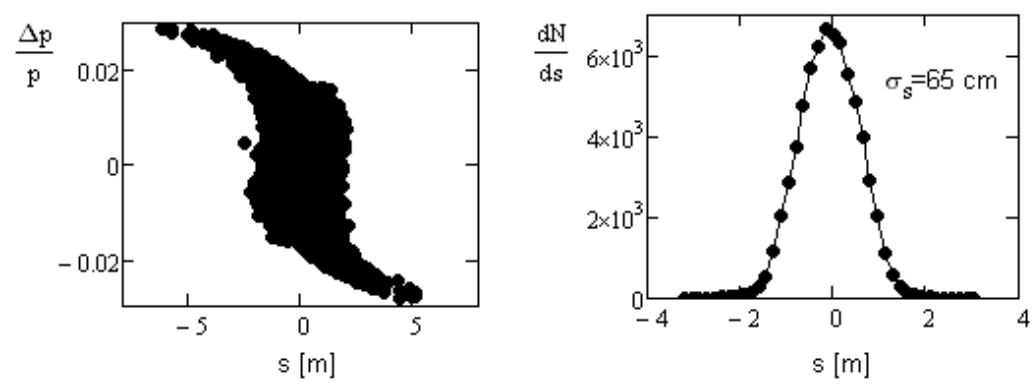

Figure 3. Results of numerical simulations of bunch compression: left - macro-particle positions in the phase space after bunch compression, right - corresponding longitudinal particle density. 


\section{Discussion}

As can b e se en $\mathrm{f}$ rom Tab le 1 both th e micr owave in stability and th $\mathrm{e}$ betatron tune shift due to beam space charge limit the beam power to $\sim 1 \mathrm{MW}$ for $8 \mathrm{GeV}$ muon collider proton driver if a single bunch at $15 \mathrm{~Hz}$ is used. B ut both limitations diminish quickly with be am energy and become insignificant above $\sim 16 \mathrm{GeV}$. Thus there are two ways to achieve $4 \mathrm{MW}$ power. The first one is an energy increase and the second one is an increase of number of bunche s and merging them on the target [5]. Both possibilities are feasible. An accurate engineering analysis is required to make the final choice.

\section{Acknowledgments}

The author would like to thank S. Nagaitsev, C. Ankenbrandt, R. Palmer and M. Popovic for useful discussions devoted to different aspects of the muon collider proton driver.

\section{References}

1. R. B. Palmer, "Muon Collider," This conference.

2. R . Garoby, et.al., "Linac-based proton driver for a neutrino factory, " This conference.

3. A. Hofmann, “Landau damping,", in "CERN Accel. School," CERN 95-06, 275 (1995).

4. M . S. Zism an, "P roton beam requirements for a neutrino factory and muon collider, " This conference.

5. R. B. Palmer, "Proton bunching options," This conference. 